\title{
Die Familie im Leben und in den Schriften der Heiligen Theresia Benedikta vom Kreuz (Edith Stein)
}

\section{Family in the Life and Works of Saint Teresa Benedicta of the Cross (Edith Stein)}

\begin{abstract}
In modern times as never before, marriage and family are in danger. The contemporary problems were already present in many aspects in the times of Edith Stein. The author of the article studies the life and the works of the Saint with regard to marriage and family. For Edith Stein, marriage is an inseparable union of a man and woman, which, thanks to the sacramental grace, is filled with special strength to maintain reciprocal love and fidelity and also to cooperate with God in giving life and educating children. In Edith Stein's opinion, both the husband and the wife in the family have rights and duties connected with being God's image, bearing and bringing up children and transforming the world by their work. Edith, however, noted the difference between sexes and the way in which the tasks were realised according to the nature and the different callings. The Saint saw the necessity of complementarity of sexes and generations in a harmonious family. Such a vision of marriage and family is still applicable in our times.
\end{abstract}

Keywords

Family, marriage, Edith Stein, Teresa Benedicta. 


\section{Einführung}

Was zur Familie gehört, wird durch der Normenkomplexe - Ehe, Elternschaft und Verwandtschaft - geregelt. ${ }^{1}$ Wie Hl. Johannes Paul II. lehrte: „Die von der Liebe begründete und beseelte Familie ist eine Gemeinschaft von Personen: des Ehemanns und der Ehefrau, der Eltern und der Kinder, der Verwandten. Ihre erste Aufgabe ist es, die Wirklichkeit ihrer Einheit treu zu leben in dem ständigen Bemühen, eine echte Gemeinschaft von Personen zu bilden“. ${ }^{2}$ Die innige Gemeinschaft des ehelichen und familiären Lebens und der Liebe ist begründet in Gott, der sie mit eigenen Strukturen ausstattet. ${ }^{3}$ Die obengenannten Voraussetzungen der katholischen Vorstellung einer Familie sollten einen Ausgangspunkt bei Forschungen über ihre Auffassung bei Edith Stein, der späteren Theresia Benedikta vom Kreuz bilden. Auch bei ihr sind die Verhältnisse von zwischenpersönlichen Bindungen von fundamentaler Bedeutung. Man sollte sie zuerst aus der biografischen Perspektive präsentieren, demnach wird man besser die Ideen verstehen können, die in den Schriften der Heiligen enthalten sind. Aufgrund der persönlichen Erfahrungen der heiligen Karmeliterin wird es einfacher, ihre Anschauungen zur Natur der Ehe und Familie und ihre fundamentalen Aufgaben zu präsentieren.

\section{Die Familie im Leben von Edith Stein}

Die Bindung von Edith Stein an ihre eigene Großfamilie musste für sie eine wesentliche Rolle spielen, wenn sie einen großen Teil ihrer autobiographischen Erinnerungen im Buch „Aus dem Leben einer jüdischen Familie“ gerade ihrer Familie widmete. ${ }^{4}$ Die Bindung an die Familie wird in ihrem Leben ein Teil der Zugehörigkeit zum Auserwählten Volk und zu der jüdischen Kultur. Es hatte für sie eine so große Bedeutung, dass sie während ihrer Verhaftung 1942 zu ihrer

${ }^{1}$ Vgl. K. S. Frank, Familie. III. Soziologisch, in: Lexikon für Theologie und Kirche, B. 3, Freiburg 2006, S. 1169.

${ }^{2}$ Johannes Paul II., Apostolisches Schreiben Familiaris consortio 18.

${ }^{3}$ Vgl. H.-J. Sander, Theologischer Kommentar zur Pastoralkonstitution über die Kirche in der Welt von heute Gaudium et spes, in: Herders Theologischer Kommentar zum Zweiten Vatikanischen Konzil, B. 4, Freiburg 2005, S. 772.

${ }^{4}$ Vgl. E. Stein, Aus dem Leben einer jüdischen Familie, Freiburg 1985. 
Schwester Rosa sagte: „Komm, wir gehen für unser Volk”. ${ }^{5}$ Die Erinnerungen von Edith Stein gehen in die Zeiten ihrer Urgroßeltern, Burchard und Courant, zurück, die von ihr als tief religiöse, arbeitsame und den Armen helfende Personen dargestellt werden. ${ }^{6}$

Eine besondere Bedeutung spielte in der Familie die Mutter von Edith, Augusta Stein, besonders nach dem Tod ihres Mannes, Siegfried. ${ }^{7}$ Sie brachte elf Kinder zur Welt, davon starben vier in der Kindheit. Sie kümmerte sich hauptsächlich um die Familie und den Haushalt, besonders um den Holzhandel, womit sie sich nach dem Tod ihres Mannes beschäftigen musste. ${ }^{8}$ In der Erinnerung der Heiligen blieb die besondere Sorge der Mutter um die Personen, die Hilfe brauchten, sowohl die ältesten als auch die jüngsten in der Familie. ${ }^{9}$ Als die Kinder schon erwachsen waren, betrachteten sie ihre Mutter als Familienoberhaupt, denn sie wussten, dass sie mit ihrer Hilfe rechnen können und ihre Meinung war für sie wichtig. ${ }^{10}$ Obwohl Augusta ihre Kinder zum einfachen und strengen Leben erzog, ließ sie ihnen viel Freiheit bei der Wahl des Lebensweges. So geschah es im Fall von Elsa, die den Beruf der Lehrerin wählte und von Edith, die das philosophische Studium begann. ${ }^{11}$ Die einzige Ausnahme bildeten die religiösen Angelegenheiten. Obwohl Augusta sich sehr bemühte ein vorbildliches, frommes und mit Gebeten erfülltes Leben zu führen, ist ihr die religiöse Erziehung der Kinder nicht gelungen. ${ }^{12}$ Aufgrund der religiösen Unterschiede kam es nicht zur Heirat von Elsa mit einem Katholiken. ${ }^{13}$ Auch Edith traute sich nicht, die Mutter über ihren Übertritt zum Katholizismus zu informieren. Denn es bedeutete für Augusta ein Zeichen der Untreue gegenüber dem einzigen Gott - Jahwe. ${ }^{14}$ Auch aus diesem Grund hielt man bis zu ihrem

${ }^{5}$ Vgl. R. Leuven, Heil im Unheil, Freiburg 1983, S. 166.

${ }^{6}$ Vgl. E. Stein, Aus dem Leben..., S. 4-15.

7 Vgl. E. Stein, Aus dem Leben..., S. $14 \mathrm{f}$.

${ }^{8}$ Vgl. E. Stein, Aus dem Leben..., S. 14-17. 33.

9 Vgl. E. Stein, Aus dem Leben..., S. 10f. 61.

10 Vgl. E. Stein, Aus dem Leben..., S. 20-24.

11 Vgl. E. Stein, Aus dem Leben..., S. 18f. 144.

12 Vgl. R. Leuven, Heil im Unheil, S. 35.

${ }^{13}$ Vgl. E. Stein, Aus dem Leben..., S. 34f.

${ }^{14}$ Vgl. E. Biberstein - Stein, Aufzeichnungen, in: E. Stein, Aus dem Leben..., S. 18f; R. Leuven, Heil im Unheil, zit. Werk, S. 48f. 
Tode geheim, dass die zweite Tochter Rosa sich zum Katholizismus bekehrte und ihre Taufe aus Rücksicht auf die Mutter verschob. ${ }^{15}$

Gerade die Geschwister spielten, neben der Mutter, eine große Rolle im Leben von Edith Stein. Als das siebte und jüngste Kind war sie besonders stark mit Erna verbunden, die im ähnlichen Alter war. Besonders nach dem Tod des Vaters, als die Mutter mehr Zeit den beruflichen Angelegenheiten opfern musste, hatte das ältere Geschwister einen großen Einfluss auf die Erziehung der Heiligen. Mit besonderer Liebe wurde sie von ihrem Bruder Paul umgeben. ${ }^{16}$ Jedoch war der Einfluss der älteren Geschwister nicht immer gut, wenn z.B. während der jüdischen Feste zu Hause, die Brüder nicht immer ernst und fromm blieben. ${ }^{17}$ Es sollte auch hinzufügt werden, dass gerade der Einfluss der älteren, ungläubigen Schwester Elsa, und insbesondere der Aufenthalt in ihrem Haus in Hamburg, dazu beitrug, dass Edith die Gebetspraxis aufgegeben hat. ${ }^{18}$ Nach der Bekehrung beklagte sich Edith nicht über die Fremdheit der Geschwister. Sie gab nur zu, dass obwohl sie sich das allerbeste für die Familie wünschte und sich als beste Tochter, Schwester und Tante verhielt, es jedoch die Verwandten ganz anders erlebten. ${ }^{19}$

In den Lebensplänen von Edith Stein spielte die Frage der Ehe und der Familiengründung eine wesentliche Rolle. Zwar deklarierte sie einerseits in ihrer frühen Jugend, von feministischen Ideen beeinflusst, dass sie nie die Berufsarbeit für die Familie opfern würde, dachte sie andererseits im Grunde des Herzens an eine Heirat. ${ }^{20}$ Sie wurde zwar nicht praktisch vorbereitet, um einen Haushalt zu führen, jedoch laut der Ehefrau von Edmund Husserl, war sie eine nützliche Hilfe im Haushalt. ${ }^{21}$ In einem der Briefe an Erna gab sie zu, dass die finanziellen Angelegenheiten sie nie bei einer Heirat stören würden. ${ }^{22}$ Gerade in dieser Zeit verrät der Briefwechsel mit dem polnischen Philosophen Roman Ingarden eine tiefe Freundschaft, die sich im Fall der Heiligen in eine unerwiderte Liebe umwandelte. Die Beruhigung kam erst 1919, als Ingarden heiratete und Edith

\footnotetext{
15 Vgl. E. Stein, Selbstbildnis in Briefen 1, Freiburg 1998, S. 220. $237 f$.

16 Vgl. E. Biberstein - Stein, Aufzeichnungen, S. $15 \mathrm{f}$.

17 Vgl. E. Stein, Aus dem Leben..., S. 44f.

18 Vgl. E. Biberstein - Stein, Aufzeichnungen, S. 16f; E. Stein, Aus dem Leben ..., S. 111. 121.

19 Vgl. E. Stein, Briefe an Roman Ingarden, Freiburg 1991, S. 203.

20 Vgl. E. Stein, Aus dem Leben..., S. 96. 136.

${ }^{21}$ Vgl. E. Stein, Briefe an Roman Ingarden, S. $107 \mathrm{f}$.

${ }^{22}$ Vgl. E. Stein, Selbstbildnis in Briefen 1, S. 31f.
} 
sich tiefer in die religiöse Entfaltung engagierte. In dieser Zeit kommt es auch $\mathrm{zu}$ einem neuen, tiefen und wieder einseitigen Verlieben in Hans Lipps. ${ }^{23}$ Die Freundschaft mit Ingarden überstand viele Jahre und Edith interessierte sich für sein Familienleben. Indem sie ihm z.B. einen Vorwurf daraus machte, dass er sie über die Geburt seines Kindes nicht informierte, vermutete sie, dass es in seiner Meinung für sie „zu menschlich“ wäre. Unterdessen betonte sie, dass sie sich auf diesem Gebiet auskenne, denn sie beschäftige sich doch ständig mit ihren Neffen. ${ }^{24}$ In den zahlreichen Ratschlägen, die sie ihren jungen Zöglingen erteilte, lässt sich einerseits eine große Achtung für die Ehe bemerken und andererseits eine Sorge um das Erwachen der Berufung zum Ordensleben. Ihrer Meinung nach sollte die deutlich erkannte Ordensberufung ihren Ausdruck im äußeren Verhalten und sogar in der Kleidung finden. ${ }^{25}$ Das führt uns zu der letzten Frage, die mit dem Leben der Heiligen verbunden ist, nämlich zum Verhältnis der natürlichen Familie zu der Ordensfamilie.

Als Edith Stein in den Karmel eintrat, betonte sie den positiven Aspekt, d.h. das Bündnis mit Christus in ständiger Gemeinschaft der Liebe, und nicht den negativen Aspekt, den der Verzicht auf die Ehe bildete. ${ }^{26}$ Ähnlich behandelte sie die familiären Bindungen und unterstrich, dass ihr Aufenthalt im Kloster keinen Verlust für die Familie, sondern einen Gewinn und eine Quelle der Gnade bedeutete. ${ }^{27}$ Die größte Quelle der Leiden war für Theresia Benedikta die physische und besonders die geistige Trennung von ihrer Mutter, für die der Eintritt der Tochter ins Kloster als die schlimmste Lösung erschien. ${ }^{28}$ Nach dem Eintritt ins Kloster traf Theresia Benedikta ihre Mutter nie wieder, die 1936 starb und sich bis zum Tode mit dem Entschluss ihrer Tochter nicht abgefunden hat. ${ }^{29}$ Einen Trost bedeutete für die Heilige der Wunsch nach Nachahmung der biblischen Esther, die aus dem Volk genommen wurde, um für das Volk zu stehen und sich vor dem König für ihr Volk einzusetzen. ${ }^{30}$

${ }^{23}$ Vgl. E. Stein, Briefe an Roman Ingarden, S. 166; H. - B. Gerl, Einleitung, in: E. Stein, Briefe an Roman Ingarden, S. 11f.

${ }^{24}$ Vgl. E. Stein, Briefe an Roman Ingarden, S. 142. 170.

25 Vgl. E. Stein, Selbstbildnis in Briefen 1, S. 187. 205f. $208 f .288$.

${ }^{26}$ Vgl. R. Leuven, Heil im Unheil, zit. Werk, S. 125.

${ }^{27}$ Vgl. E. Stein, Briefe an Roman Ingarden, S. 235.

28 Vgl. E. Stein, Briefe an Roman Ingarden, S. 234.

29 Vgl. E. Stein, Selbstbildnis in Briefen 2, Freiburg 1977, S. 59-63. 68n.

${ }^{30}$ Vgl. E. Stein, Selbstbildnis in Briefen 2, S. 121. 
Gemeinsam mit ihrer Schwester Rosa versprach sie lebenslang für die Eltern zu beten. ${ }^{31}$ Der Tod der Mutter eröffnete Rosa die Möglichkeit der Taufe und vertiefte die Bindung zwischen den Schwestern. ${ }^{32}$ Und auf diese Weise konnte Edith auch im Karmel sich um die Verbundenheit mit der Familie kümmern, wovon der Wunsch zeugt, in einem in Breslau geplanten Kloster zu wohnen. ${ }^{33}$ Es ändert aber nichts daran, dass ihr Herz sich von Gottes Liebe zu der neuen Ordensfamilie erfüllte. Wie es eine Protestantin - Hedwig Conrad-Martius, die sie im Karmel besuchte, feststellte, zu Theresia Benedikta passe die doppelte Bedeutung des Wortes "gratia“ gut, das sowohl die Gnade als auch die Anmut bedeute. ${ }^{34}$ Bezeichnend ist das Geständnis der Heiligen anlässlich der ersten Gelübde 1935, dass sie im größeren Teil ihres Lebens mehr einsam war als jetzt im Kloster. Im Karmel fehlte ihr nichts von der Außenwelt, und zugleich hatte sie alles, was ihr fehlte, als sie in der Welt lebte. ${ }^{35}$ Es heißt aber nicht, dass sie ihre Geschwister vergaß, sie interessierte sich beispielsweise für das Schicksal ihrer Schwester in New York. ${ }^{36}$

\section{Die Auffassung der Ehe und Familie}

Die Ehe bedeutete für Edith Stein die Grundlage der Familie, die Ehe, das heißt die Beziehung zwischen dem Mann und der Frau. Im Gegensatz zu den feministischen Tendenzen betonte die Heilige die Geschlechterunterschiede, die sowohl den Körper als auch die Seele betreffen. ${ }^{37}$ Sie meinte, der Frau entspreche eher die Einheit und Kompaktheit der ganzen körperlich-geistigen Struktur und eine harmonische Entwicklung; dem Mann dagegen die Verstärkung der einzelnen Kräfte bis zum Erreichen der höchsten Leistungen. Edith sah diese dem Menschen gegebenen und aufgegebenen Unterschiede im Buch Genesis.

31 Vgl. E. Stein, Selbstbildnis in Briefen 2, S. 65. 120.

32 Vgl. E. Stein, Selbstbildnis in Briefen 2, S. 71. 73. 80.

${ }^{33}$ Vgl. E. Stein, Selbstbildnis in Briefen 1, S. 328.

${ }^{34}$ Vgl. A. U. Müller, M. A. Neyer, Edith Stein. Das Leben einer ungewöhnlichen Frau, Zürich 1998, S. 235.

${ }^{35}$ Vgl. E. Stein, Selbstbildnis in Briefen 2, S. 30.

${ }^{36}$ Vgl. E. Stein, Selbstbildnis in Briefen 2, S. 144.

${ }^{37}$ Vgl. E. Stein, Probleme der Frauenbildung, in: Edith Steins Werke, B. 5, Die Frau. Ihre Aufgabe nach Natur und Gnade, Freiburg 1959, S. 131-133. 
Dort werden der Mann und die Frau dazu berufen, die Erde zu beherrschen, das heißt ihre Dinge zu erkennen, sich ihrer zu erfreuen und sie schöpferisch zu verwandeln. Dies bedeutet die grundsätzliche Aufgabe des Mannes, an dessen Seite die Frau, als Gefährtin steht. Der Mann und die Frau werden dazu berufen, den Nachwuchs zu erzeugen und zu erziehen. Da die Frau körperlich und seelisch stärker mit dem Kind verbunden ist, erkennt sie darin ihre Hauptaufgabe. An ihrer Seite steht der Mann als Hilfe und Schutz. Der Mann und die Frau sollen schließlich das Abbild Gottes sein. Auch diese Aufgabe sollten sie auf eine spezifische Weise erfüllen. Die Frau verwirklicht diese Berufung durch eine harmonische Entwicklung der Kräfte und der Mann durch stärkere Entwicklung der einzelnen, in ihm steckenden Möglichkeiten. Das alles sollte sowohl im Kontext der gemeinsamen menschlichen Natur, als auch im Kontext der individuellen Prädisposition jedes Individuums betrachtet werden. ${ }^{38}$

In einer eigenartigen theologischen Vorstellung des Menschen präsentiert sie ihn aus der Perspektive der Heilsgeschichte. Sie betont die ursprüngliche Einheit der beiden, Freiheit von der Sünde, Bewahren vor Leiden, Tod und Gier und das Hervorheben zur Würde das Kind Gottes zu sein. ${ }^{39}$ Die Unterschiede äußern sich darin, dass von Anfang an die Frau als Gefährtin des Mannes und Mutter berufen wird. ${ }^{40}$ Das bedeutet, dass ihr besonderes Glück in der Teilnahme am Leben des anderen Menschen besteht, währenddessen den Mann „seine Angelegenheiten“ in Anspruch nehmen. ${ }^{41}$ Gemeinsam sollten "die zwei ein Fleisch sein“. Edith meint, dass die Beziehung der Frau zum Mann, nach den Plänen des Schöpfers, nicht nur körperlich sein konnte. Gott hat beide als geistige,

38 „Mann und Frau sind bestimmt, die Erde zu beherrschen, d. h. die Dinge dieser Welt zu erkennen, sich daran zu freuen und sie in schöpferischer Tat zu gestalten. Aber dem Mann sind diese Kulturleistungen als erste Aufgabe zugewiesen, die Frau ist ihm als Gehilfin zur Seite gestellt. Mann und Frau sind bestimmt, Nachkommenschaft zu erzeugen und zu erziehen. Aber die Frau, die mit dem Kinde leiblich und seelisch stärker verbunden und durch diese Verbindung in ihrer ganzen Lebensgestaltung enger gebunden ist, hat darin ihre erste Aufgabe (...). Mann und Frau ist es aufgegeben, Gottes Bild in sich auszuprägen. In der Endlichkeit des ihnen mitgeteilten Seinsmaßes liegt es, dass sie auch das auf spezifische Weise tun müssen. (...) die Frau mehr durch harmonische Entfaltung aller Kräfte, der Mann durch stärkere Entfaltung einzelner“. E. Stein, Probleme der Frauenbildung, S. 138f.

39 Vgl. E. Stein, Was ist der Mensch? Eine theologische Anthropologie, Freiburg 1994, S. $26 \mathrm{f}$. 35-37.

${ }^{40}$ Vgl. E. Stein, Der Eigenwert der Frau in seiner Bedeutung für das Leben des Volkes, in: Edith Steins Werke, B. 5, Die Frau. Ihre Aufgabe nach Natur und Gnade, Freiburg 1959, S. $208 \mathrm{f}$.

${ }^{41}$ Vgl. E. Stein, Das Ethos der Frauenberufe, in: Edith Steins Werke, B. 5, Die Frau. Ihre Aufgabe nach Natur und Gnade, Freiburg 1959, S. 3f. 
persönliche Wesen geschaffen, die zur Einheit in gegenseitiger Liebe berufen wurden. Gott hat die Frau als ähnlich, aber doch verschieden geschaffen, damit sie den Geist und den Leib des Mannes ergänzen konnte. ${ }^{42}$ Jedoch aufgrund der Erbsünde der ersten Menschen kam es zur Störung der Beziehung zu Gott, zum Menschen, zum Nachwuchs, zur ganzen Schöpfung und sogar zu sich selbst. ${ }^{43}$ Erst das Reich Gottes, von Christus gebracht, schuf eine neue Ordnung in der Beziehung der Geschlechter durch die Ablehnung dessen, was Folge der Sünde war, und durch die erneute Einführung der ursprünglichen Ordnung (vgl. Mt 19, 1-12; Mk 10, 1-12). Neben der Betonung der Unauflöslichkeit der Ehe führte Christus auch das Ideal der Jungfräulichkeit ein. ${ }^{44}$ Als Krönung der Lehre von Edith Stein über die Beziehung zwischen dem Mann und der Frau gilt der vom heiligen Paulus (Eph 5) entnommene Vergleich dieser Bindung mit der Beziehung von Christus und der Kirche. ${ }^{45}$

In der synthetischen Auffassung stellt Edith die Lehre der Bibel zum Thema der Beziehung zwischen Mann und Frau dar. Sie meint, dass beide unterschiedliche Berufungen haben, nach der ursprünglichen Ordnung und im Zustand der gefallenen Natur und der Erlösung. Nach dem Sündenfall wird das gegenseitige Verhältnis der reinen Liebesgemeinschaft durch die Gier gestört und es verwandelt sich in eine Herrschaft. Der Mann wird zum schweren Kampf ums Dasein verurteilt und die Frau zum Mühsal der Geburt. Das Heilsversprechen beruht darauf, dass der Frau der Kampf mit dem Bösen anvertraut wurde, und der Mann die Vervollkommnung im zukünftigen Menschensohn findet. Die Erlösung hat zum Ziel das Wiederherstellen der ursprünglichen Ordnung. Der Vorzug des Mannes wird dadurch zum Ausdruck gebracht, dass der Erlöser in der Gestalt eines Mannes zur Welt kommt. Das weibliche Geschlecht wird auf diese Weise geehrt, dass der Erlöser von einer menschlichen Mutter geboren wird, die ein Übergangstor von Gott zum menschlichen Geschlecht bildete. Ähnlich wie Adam - Urbild des Menschen, der auf den zukünftigen göttlich menschlichen König der Geschöpfe deutete, so sollte auch jeder Mann im Reich Gottes in der ehelichen Gemeinschaft die liebende Sorge Christus um die Kirche abbilden. Die Frau dagegen sollte in einer freien und liebevollen Hingabe im

${ }^{42}$ Vgl. E. Stein, Endliches und ewiges Sein, Freiburg 1986, S. 469-471.

${ }^{43}$ Vgl. E. Stein, Beruf des Mannes und der Frau nach Natur - und Gnadenordnung, in: Edith Steins Werke, B. 5, Die Frau. Ihre Aufgabe nach Natur und Gnade, Freiburg 1959, S. 21.

${ }^{44}$ Vgl. E. Stein, Beruf des Mannes und der Frau..., S. $24 f$.

${ }^{45}$ Vgl. E. Stein, Beruf des Mannes und der Frau..., S. $25 f$. 
Mann das Abbild Christi ehren und selbst zum Abbild der Muttergottes, also auch zum Abbild Christi werden. ${ }^{46}$

Edith baute ihre Ehevorstellung auf der Grundlage der Bibel, und vor der Bekehrung wich sie nicht grundsätzlich von den Ideen, die sie später im Christentum fand, ab. Auch in ihrer jüdischen Familie bildete die Unauflöslichkeit der Ehe einen wesentlichen Wert. ${ }^{47}$ Mittlerweile konnte man in der Gesellschaft weitgehende Veränderungen feststellen. Edith bemerkte unter der Jugend die Verkündung des Rechts auf freien Geschlechtsverkehr. Es hatte offene Beziehungen zur Folge, die als Ergebnis einer Ablehnung der traditionellen Moral galten. Wiederum strebten diese Personen, die weiter die Ehe als ein Heiligtum betrachtet haben, bewusst nach Familiengründung. Eine wirksame Antwort auf die neuen kulturellen Strömungen sah die Heilige in der katholischen Vorstellung der Ehe als ein Sakrament, dessen Ziel die Zeugung und die Erziehung des Nachwuchses sei. Da die allgegenwärtige Diskussion über Sexualität, Psychologie, Pädagogik und sogar Pathologie die Erziehungspraxis durchdringt, sollten nach Edith, die neuen Auffassungen mit der katholischen Anschauung konfrontiert werden. Es ging ihr um eine ernste und tiefe Diskussion darüber, was von den damaligen Tendenzen sich mit dem katholischen Glauben und seiner Moral vereinbaren lässt. Edith sah auch eine wichtige Aufgabe in der Bildung einer gründlichen und wirklich katholischen Theorie von Sexualität und Ehe. ${ }^{48}$

An erster Stelle, meinte die Heilige, sei die Ehe nicht nur eine natürliche menschliche Beziehung, dank derer sich das menschliche Geschlecht entwickelt, sondern vor allem ein Sakrament. Die Sakramente enthalten eine Gnade und sie wird denjenigen erteilt, die sie würdig annehmen. Die Heilige sah in der sakramentalen Ehe ein Symbol der Vereinigung von Christus und der Kirche. Sie erwähnt, gemäß der damaligen Enzykliken, dreierlei Vorteile der Ehe, d.h. den Nachwuchs, die Treue und die Unauflöslichkeit (fides, proles, sacramentum). Diese ewig geltende Unauflöslichkeit drückte Adam aus, indem er sagte, dass Eva das Bein von seinem Bein und das Fleisch von seinem Fleisch sei. Deswegen verlässt der Mann seinen Vater und seine Mutter, und die zwei werden ein Fleisch sein (vgl. Gen 2, 23f; Eph 5, 31). Christus betonte diese Einheit, indem er sagte, dass was Gott verbunden habe, dürfe der Mensch nicht trennen

\footnotetext{
${ }^{46}$ Vgl. E. Stein, Beruf des Mannes und der Frau..., S. $28 \mathrm{f}$.

${ }^{47}$ Vgl. E. Stein, Aus dem Leben..., S. 24.

${ }^{48}$ Vgl. E. Stein, Probleme der Frauenbildung, S. $96 f$.
} 
(vgl. Mt 19, 6). Die Gnade, die zur Vervollkommnung der natürlichen Liebe und zur Befestigung der unauflösbaren Einheit nötig ist, verdiente Christus den Ehegatten durch seinen Kreuzweg. Dies betonte der Hl. Paulus, indem er empfiehlt, dass die Männer ihre Frauen, wie Christus seine Kirche, für die er sich hingegeben hat, lieben (vgl. Eph 5, 25). Die Ehe bedeutet demnach eine übernatürliche Beziehung, die den Ehegatten die Gnade, besonders die Gnade der Treue erteilt. Die Unauflöslichkeit der Ehe ist so stark, dass sogar ein Verrat die Auflösung dieser Bindung nicht begründet. Edith erinnert daran, dass als Form dieses Sakraments die Zustimmung der Ehepartner gilt. ${ }^{49}$ Dank diesem Sakrament erhalten die Eltern eine spezifische, übernatürliche Ausstattung, die sie befähigt, die erhabene Berufung der Erzeugung und Erziehung des Nachwuchses, zu verwirklichen. ${ }^{50}$

Indem sich die Heilige mit der sozialen Natur des Menschen beschäftigte, erwähnte sie die familiären, freundschaftlichen, geschlechtlichen, nationalen und religiösen Gemeinschaften. ${ }^{51}$ Die Existenz als Individuum und zugleich als Gemeinschaftsmitglied begründete Edith durch die Erschaffung des Menschen als Abbild Gottes in der Heiligen Dreifaltigkeit. ${ }^{52}$ Demnach entdeckte sie überall diese eigenartige Dreifaltigkeit. ${ }^{53}$ Am wichtigsten sind jedoch ihre Betrachtungen über den vorbildlichen Charakter der Heiligen Dreifaltigkeit für die Familie selbst. Zuerst erinnert Edith Stein daran, dass sowohl der Hl. Augustin, als auch der Hl. Thomas von Aquin die Suche nach dem Abbild Gottes in der Personenmenge ablehnten. Deswegen bedeuteten zum Beispiel für sie die Dreifaltigkeit des Mannes, der Frau und des Kindes keine direkte Abbildung des innergöttlichen Lebens. Sie betrachteten demnach die Erzeugung als einen körperlichen Akt. ${ }^{54}$ Währenddessen konnte, laut der Heiligen, in diesem Akt das Abbild der Heiligen Dreifaltigkeit gesehen werden, unter der Bedingung,

49 Vgl. E. Stein, Was ist der Mensch?..., S. 116f. 162f. 165. 172.

50 Vgl. E. Stein, Ganzheitliches Leben. Schriften zur religiösen Bildung, Edith Steins Werke, B. 12, Freiburg 1990, S. 223.

${ }^{51}$ Vgl. E. Stein, Eine Untersuchung über den Staat, „Jahrbuch für Philosophie und phänomenologische Forschung" 7, Halle a. d. S. 1925, S. 4; E. Stein, Beiträge zur philosophischen Begründung der Psychologie und der Geisteswissenschaften. Individuum und Gemeinschaft, "Jahrbuch für Philosophie und phänomenologische Forschung" 5, Halle a. d. S. 1922, S. 175.

52 Vgl. E. Stein, Die theoretischen Grundlagen der sozialen Bildungsarbeit, in: Edith Stein Gesamtausgabe, B. 16, Freiburg im Breisgau 2001, S. 18.

${ }^{53}$ Vgl. E. Stein, Endliches und ewiges Sein..., S. 249.

${ }^{54}$ Vgl. E. Stein, Endliches und ewiges Sein..., S. 428. 
dass man ihn als einen freien, geistigen Akt der Hingabe betrachtet. Tatsächlich finden wir bei Edith Stein Versuche derartiger Interpretation in den früheren Arbeiten, indem sie über die Heilige Dreifaltigkeit schrieb und feststellte, dass so wie der Sohn von Vater stammt, und der Geist von beiden, so stammt die Frau vom Mann, und von beiden stammt der Nachwuchs. ${ }^{55}$ Dies bildete für Edith Stein die tiefste Quelle der Würde der menschlichen Familie.

Die Heilige betonte, gemäß der Kirchenlehre, den großen Wert der Ehe. Gott schuf alle Menschen und hat sie zur ewigen Glückseligkeit berufen, und die Geschlechterbeziehung wirkt bei der Erschaffung und Heiligung mit. Im Angesicht dessen muss diese Beziehung etwas Großes, Heiliges und Wesentliches im Bauen und Leben der Kirche bedeuten. Zur Vollständigkeit dieses Bildes sollte noch hinzugefügt werden, dass die heilige Karmeliterin meinte, die Ehe sei keine Pflicht, da die Jungfräulichkeit etwas Höheres bedeutet. ${ }^{56}$ Das Abbild Gottes zu sein, laut Edith Stein, erfolge nicht nur durch die Ehe und die Erzeugung des Nachwuchses. Natürlich wurden in Israel die gebärenden Frauen gesegnet (besonders diejenigen Frauen, die Söhne bekamen) und die Unfruchtbaren verflucht. Im 113. Psalm wird als besonderer Ausdruck der Güte Gottes die unfruchtbare Frau zur Mutter. Die Mutter und Ehefrau genoss eine große Achtung in Israel. Ihre Aufgaben bestanden nicht nur im Gebären des Nachwuchses und in der Sorge um seine körperliche Entwicklung, sondern auch in der Erziehung in Gottesfurcht. Der Kampf mit dem Bösen war ihre große Berufung, bis auf die Mutter des Sohnes, der den Tod und die Hölle besiegte. An der Schwelle der Testamente steht, neben dem neuen Adam auch die neue Eva, als ausdrücklichster Beweis der ewigen Bedeutung und der ewigen Werte der Geschlechterunterschiede. Maria war entschieden in einer Freiheit von der körperlichen Beziehung zu leben. Sie war dem Mann gehorsam, der ihr als Beschützer gestellt wurde, aber sie vereinte sich nicht körperlich mit ihm und die Erzeugung des Nachwuchses bildete nicht den Sinn ihrer Ehe. In Maria bekam die Menschheit das Abbild der jungfräulichen Reinheit. Sowohl Jesus, als auch Maria stammen dem menschlichen Geschlecht ab, aber sie sind von allen Bindungen frei, die eine Erfüllung des menschlichen Lebenssinnes nur von der Vereinigung mit einem Menschen abhängig machen. Bei ihnen

55 „Nach dem Bilde Gottes schuf Gott den Menschen. Gott aber ist dreieinig: wie aus dem Vater der Sohn hervorgeht und aus Sohn und Vater der Geist, so ist das Weib vom Mann ausgegangen und von ihnen beiden Nachkommenschaft". E. Stein, Beruf des Mannes und der Frau..., S. 20.

${ }^{56}$ Vgl. E. Stein, Was ist der Mensch?..., S. 173f. 
tritt anstelle dieser Bindung die Vereinigung mit Gott, bei Christus durch die hypostatische Union, und bei Maria durch die Hingabe des ganzen Daseins in Dienste Gottes. ${ }^{57}$

\section{Die Aufgaben der Eltern}

Die oben angedeutete Auffassung der Ehe und Familie bildet in den Schriften von Edith Stein eine Grundlage, um die Aufgaben der Eltern vorzuzeigen. Denn nach ihrer Auffassung ist die Berufung des Menschen schon in bestimmter Weise in seiner Natur vermerkt, aus esse sollte das agere kommen. Dazu kommt noch das wesentliche Ausmaß der göttlichen Aufforderung, woran an dieser Stelle nochmals erinnert werden sollte. Gott vertraute dem Mann und der Frau eine gemeinsame Berufung an (vgl. Gen 1, 26-29). Sie sollen, jeder auf eigene Weise, ein Abbild Gottes sein, die Erde bevölkern und sich die Erde unterwerfen ${ }^{58}$. Jede dieser Aufgaben, mit Berücksichtigung der Geschlechterunterschiede, wirkt aufeinander und beeinflusst das Funktionieren einer Familie.

An die erste Stelle rückt die Aufgabe Abbild Gottes zu sein. Wie schon erwähnt wurde, verwirklicht die Frau diese Aufgabe durch eine harmonische Entwicklung, der Mann dagegen durch größere Konzentration auf die einzelnen Möglichkeiten. Gerade in dieser Aufgabe sollten sich die Ehepartner gegenseitig unterstützen und ergänzen. Ihre gegenseitige Hilfe wird durch die Verwirklichung von zwei weiteren Berufungen erfüllt, deswegen sollte man sie zuerst aus der Perspektive der ehelichen Beziehungen betrachten. Edith Stein meint, es sei wichtig, dass die Frau an der Hauptaufgabe des Mannes teilnehme, die auf der Beherrschung der Erde beruhe. Deswegen erklärt sich auch ihr großes Engagement für eine vielseitige Ausbildung der Frauen und deren Zugang zu der Kulturwelt. Indem der Ehemann der Frau die Möglichkeit der Teilnahme an seinen Aktivitäten entzieht und sie in der Entwicklung begrenzen will, wird er verantwortlich für die Unterdrückung ihres geistigen Lebens und für eine zu starke Bindung an die Familie. Wie die Heilige meint, sei der Ehemann als Familienoberhaupt auch für ihr übernatürliches Leben verantwortlich. Diese Aufgabe wäre zu schwer, wenn an seiner Seite nicht die Frau stünde. Denn vor allem in ihr besteht das Streben

\footnotetext{
57 Vgl. E. Stein, Probleme der Frauenbildung, S. 148-150.

${ }^{58}$ Vgl. E. Stein, Beruf des Mannes und der Frau..., S. 18-20; E. Stein, Probleme der Frauenbildung, S. 138f.
} 
nach der unbeschränkten und integralen Entwicklung der eigenen und fremden Persönlichkeit. Gerade die Frau charakterisiert eine besondere Empfindlichkeit für Gottes Angelegenheiten und für die ästhetische und moralische Schönheit, und dank diesen Eigenschaften kann sie den Mann vor dem Vernachlässigen dieser Lebensdimensionen, zugunsten einseitiger Widmung aller Kräfte der Berufstätigkeit, schützen. ${ }^{59}$

Demnach wird der Mann, durch die harmonische Entfaltung der Kräfte der Frau, von übermäßiger Einseitigkeit bewahrt. Und wenn der Mann und die Frau dazu geschaffen worden sind, um sich gegenseitig zu ergänzen, wird dieses Bedürfnis noch stärker gegenüber dem Nachwuchs auftreten. Die nahe körperliche Beziehung mit der Mutter, die besondere Veranlagung der Frau zur Teilnahme am Leben und zum Dienst im Leben, und die natürliche Neigung zur harmonischen Entwicklung der Kräfte deuten darauf, dass die Hauptbeteiligung an der Erziehung zu ihr gehört. Andererseits erfordern die mütterlichen Aufgaben einen Schutz von der Seite des Mannes. Er trägt auch eine bestimmte Aufgabe, den Nachwuchs in das Erwerben besonderer Lebensleistungen einzuführen. Letztendlich legt die Aufgabe der Beherrschung der Geschöpfe ihm die Sorge um den Nachwuchs auf. Es sollte auch daran erinnert werden, dass nicht nur der Mann und die Frau zur gegenseitigen Ergänzung berufen sind, sondern dass auch die nachfolgenden Generationen ein Recht darauf haben, das Eigene und Neue beizutragen. Zum Erziehungswesen gehört demnach die Achtung dessen, was das Neue und Eigene in der jungen Generation bedeutet. ${ }^{60}$

Obwohl Edith Stein an die erzieherischen Aufgaben des Vaters erinnerte, widmete sie besonders viel Platz der Beziehung des Kindes zu der Mutter. Die Bindung und den Einfluss der Mutter auf das Kind schilderte die Heilige schon in der pränatalen Phase. ${ }^{61}$ Auch nach der Geburt bleibt eine unsichtbare Bindung, dadurch fühlt die Mutter, was das Kind braucht. Deswegen ist ihre Rolle unersetzbar und das Kind wird sich ohne die Mutter nicht auf diese Weise entfalten, wie wenn es von ihrer Liebe umgeben ist. Ihre Hauptaufgabe besteht

\footnotetext{
59 Vgl. E. Stein, Beruf des Mannes und der Frau..., S. 36f.

60 „Dabei ist zu berücksichtigen, dass nicht nur Mann und Frau zur gegenseitigen Ergänzung geschaffen sind, sondern auch die Folge der Generationen, so dass jede folgende berufen ist, Neues und Eigenes zu verkörpern und zu leisten, und die ehrfürchtige Berücksichtigung dessen, was Neues und Eigenes in der jungen Generation heranwachsen will, gehört wesentlich zur Erziehung“. E. Stein, Beruf des Mannes und der Frau..., S. $31 \mathrm{f}$.

${ }^{61}$ Vgl. E. Stein, Mütterliche Erziehungskunst, in: Edith Stein Gesamtausgabe, B. 13, Freiburg 2002, S. 116; E. Stein, Endliches und ewiges Sein..., S. 471f.
} 
im Zusammensein mit dem Kind, mit dem Bewusstsein, dass es kein Spielzeug ist, welches ihre mütterlichen Bedürfnisse befriedigt, aber eine Gottesgabe, deren Natur durch das Einschließen in die Gesellschaft entwickelt werden sollte. Von Anfang an sollte die Mutter ihr Kind vor den schädlichen Einflüssen der Außenwelt schützen. ${ }^{62}$ Sie sollte es zu Gehorsam, Reinheit, Regelmäßigkeit und Zähmen der Triebe erziehen, und ihm dem Alter entsprechende Freiheiten lassen. Man solle nicht, so die Heilige, den Gehorsam in Dingen verlangen, worüber das Kind selbst entscheiden könne. Sowohl falsche Autoritäten, als auch falsche Freiheit müssen vermieden werden. ${ }^{63}$ Wichtig ist, dass das Kind, das gerade das Sprechen erlernt, zur Wahrhaftigkeit erzogen wird. Das wichtigste Erziehungsprinzip sei, laut Edith Stein, die Liebe, die man im jeden Befehl spürt und die keine Angst zulässt. Am wirksamsten ist das lebendige Beispiel, nicht das belehrende Wort. ${ }^{64}$ Eine Mutter weiß, dass sie das Kind nicht vor den Gefahren der Seele und des Körpers beschützen kann, und nicht immer bei seiner Seite stehen wird. Deswegen auch muss sie das Kind in der Bindung an Gott erziehen, damit es in ihm Hilfe und Trost findet, und sein eigenes Verhalten nach Gottes Maß misst. Wenn ihr das gelingt, kann sie ruhig um die Zukunft des Kindes bleiben. ${ }^{65}$

Wenn das Haus seine Funktionen erfüllt, indem es ein Ort ist, wo die Kinder unter Geschwistern und in der Umgebung, die an die körperlichen und geistigen Bedürfnisse angepasst ist, und unter der verantwortlichen Fürsorge der Eltern aufwachsen, findet hier die Erziehung des Menschen durch den Menschen statt. Es bedeutet ein stilles, ständiges, organisches und treues Heranwachsen, unter dem teilweise bewusst und teilweise unbewusst geführten und bildenden Einfluss der Umgebung. ${ }^{66}$ Auch die beste Familie ist nicht allen Aufgaben gewachsen, da sie nicht über alle Mittel der Selbstvervollkommnung verfügt, obwohl sie eine der besten aller erzieherischen Gemeinschaften ist. Sie muss durch andere Gemeinschaften unterstützt werden.${ }^{67}$ Deswegen auch deutete Edith Stein, neben der Familie, auf eine große Rolle der Kirche und des Staates in der Erziehung

\footnotetext{
${ }^{62}$ Vgl. E. Stein, Ganzheitliches Leben..., S. 152f.

${ }^{63}$ Vgl. E. Stein, Ganzheitliches Leben..., S. 154f.

${ }^{64}$ Vgl. E. Stein, Ganzheitliches Leben..., S. $156 f$.

${ }^{65}$ Vgl. E. Stein, Mütterliche Erziehungskunst, S. 120.

${ }^{66}$ Vgl. E. Stein, Probleme der Frauenbildung, S. $173 f$.

${ }^{67}$ Vgl. E. Stein, Probleme der Frauenbildung, S. $160 f$.
} 
des Menschen hin. Besonders viel schrieb die Heilige über die Rolle der Schule, die bei der Kindererziehung den Eltern behilflich sei. ${ }^{68}$

Die Frage des Gebärens und der Erziehung des Nachwuchses verbindet sich in der Familie mit der Frage der Vereinbarkeit der Familienpflichten mit der Berufstätigkeit, hauptsächlich der Frauen und Mütter. Edith Stein betätigte sich in ihrer Jugend aktiv in der feministischen Bewegung für den breiteren Zugang der Frauen zur Arbeitsmöglichkeit. ${ }^{69}$ Die Entwicklung auf diesem Gebiet überraschte viele unvorbereitete Frauen, die dem ruhigen häuslichen Kreis entrissen wurden und sich dadurch in verschiedenen problematischen Verhältnissen befanden. ${ }^{70}$ Wogegen anfangs sich beruflich vorwiegend diejenigen Frauen betätigten, deren natürliche Begabungen und Neigungen sie in diese Richtung lenkten, zwang die Wirtschaftskrise später viele Frauen dazu, die es nie freiwillig getan hätten. ${ }^{71}$ Mit der Zeit wurde die Berufstätigkeit der Frauen zur Notwendigkeit. Die Mädchen aus gutbürgerlichen und adligen Häusern wählten die akademischen Berufe, oft ohne entsprechende Begabungen und Neigungen. Deswegen auch kann in ihrem Fall keine Rede von der Freude über den Beruf sein. Anfangs wurde den Frauen der Zugang zu allen männlichen Berufen verweigert. Die Revolution führte zur Erfüllung fast aller Forderungen, ohne einen Vorbereitungsprozess, was viele Konflikte und eine gewisse Berufsmüdigkeit verursachte. ${ }^{72}$

In dieser Frage vertritt Edith Stein eine ausgeglichene Einstellung, die auf der Analyse der Berufung der ersten Menschen beruhte. In der Beschreibung der Welterschaffung wurde die Frau neben den Mann gestellt, damit er nicht einsam ist, und eine entsprechende Hilfe hat. Demnach erfüllt die Frau die Berufung der Ehefrau, indem sie die Angelegenheiten des Mannes zu den eigenen macht. „Seine Angelegenheiten“ bedeuten normalerweise die Berufstätigkeit. Die Teilnahme der Frau an seiner Berufstätigkeit kann vielfältig sein. Ihre erste Aufgabe besteht in der Gestaltung des Familienlebens auf eine Weise, dass es bei seiner Berufsarbeit nicht stört. Wenn die Arbeit zu Hause ausgeübt wird, sollte die

${ }^{68}$ Vgl. E. Stein, Jugendbildung im Licht des katholischen Glaubens. Bedeutung des Glaubens und der Glaubenswahrheiten für Bildungsidee und Bildungsarbeit, in: Edith Stein Gesamtausgabe, B. 16, Freiburg 2001, S. 86 f.

${ }^{69}$ Vgl. E. Stein, Selbstbildnis in Briefen 1, S. 266.

${ }^{70}$ Vgl. E. Stein, Christliches Frauenleben, in: Edith Steins Werke, B. 5, Die Frau. Ihre Aufgabe nach Natur und Gnade, Freiburg 1959, S. 45.

${ }^{71}$ Vgl. E. Stein, Christliches Frauenleben, S. 64f.

${ }^{72}$ Vgl. E. Stein, Probleme der Frauenbildung, S. $97 \mathrm{f}$. 
Frau alle Hindernisse beseitigen, die dabei stören könnten. Wenn der Mann sie außerhalb des Hauses ausübt, wird die Frau sich bemühen, ihm die verdiente Erholung zu ermöglichen. Ein unmittelbarer Einsatz und direkte Hilfe bei der Berufsarbeit des Mannes sind nur bei Personen möglich, die ähnliche Berufsausbildung oder mindestens ähnliche Interessen haben. Auf diese Weise funktionierte es oft in den Bauernfamilien, aber auch in den Häusern der Kaufmänner, Ärzte und besonders in protestantischen Gemeinden. ${ }^{73}$ Wenn die Aufgabe des Mannes im Kampf um das Dasein und die Sicherung des nötigen Unterhalts der Familie besteht, sollte dagegen die Frau vernünftig mit dem Einkommen wirtschaften, aber auch immer öfter eine Berufsarbeit aufnehmen. Darin besteht die Gefahr, dass sich die außerhäuslichen Aktivitäten häufen und es unmöglich machen werden, das Herz und Seele der Familie zu sein, was nach der Meinung der Heiligen die wesentliche Berufung der Frau für immer bleiben sollte. ${ }^{74}$ Es ändert aber nichts an der Tatsache, dass nach Edith, in Notfällen die Frau jeden Beruf ausüben kann, obwohl sie von Frauenberufen, wie dem Beruf der Ärztin, Krankenschwester, Lehrerin, Erzieherin, Sozialarbeiterin oder Haushälterin sprach. ${ }^{75}$

\section{Schlussfolgerungen}

In der gegenwärtigen Epoche sind wie nie zuvor die Ehe und die Familie bedroht. Johannes Paul II. sprach von der Brutalisierung in den Medien, in denen die Erotik, Gewalt, Apologie der Scheidungen oder antisozialer Haltungen der Jugendlichen die grundsätzlichen Werte der Familie anfechten und einen Angriff auf das wahre Wohl des Menschen bedeuten. ${ }^{76}$ Papst Franziskus schreibt in "Amoris laetitia”: „Das Wohl der Familie ist entscheidend für die Zukunft der Welt und der Kirche. Unzählige Analysen sind über die Ehe und die Familie, über ihre aktuellen Schwierigkeiten und Herausforderungen erstellt worden. Es ist heilsam, auf die konkrete Wirklichkeit zu achten, denn »die Forderungen und Anrufe des göttlichen Geistes sprechen auch aus den Ereignissen der Geschichte«, durch die »die Kirche (...) zu einer tieferen Kenntnis des unerschöpflichen Mysteriums der

\footnotetext{
${ }^{73}$ Vgl. E. Stein, Christliches Frauenleben, S. 66f.

${ }^{74}$ Vgl. E. Stein, Christliches Frauenleben, S. 68f.

${ }^{75}$ Vgl. E. Stein, Das Ethos der Frauenberufe, S. 7.

${ }^{76}$ Vgl. Johannes Paul II., Apostolisches Schreiben Familiaris consortio, 76.
} 
Ehe und Familie geführt werden kann « " ${ }^{77}$ Alle heutige Probleme der Familie traten schon in den Lebenszeiten von Edith Stein auf, die jedoch das Glück hatte, in einer Groß- und Mehrkinderfamilie aufzuwachsen, die durch tiefe Relationen verbunden war. Ihre Anschauungen zur Würde der Ehe und der Familie, die sie zu Hause erlebt hatte, erschienen als solche, die mit dem Ideal übereinstimmen, das sie in der katholischen Kirche kennen gelernt hat. Die Ehe bedeutete für sie eine unauflösliche Bindung zwischen dem Mann und der Frau, die dank der sakramentalen Gnade sich mit einer besonderen Kraft erfüllen, um die gegenseitige Liebe, Treue und Zusammenarbeit mit Gott im Erzeugen und in der Erziehung des Nachwuchses zu erhalten. Edith Stein räumte in der Familie, sowohl dem Mann als auch der Frau, die Rechte und die Pflichten ein, die damit verbunden waren, als Abbild Gottes zu sein, den Nachwuchs zu erzeugen und erziehen, und die Welt durch die Arbeit umzuwandeln. Jedoch bemerkte sie die Geschlechterunterschiede und die von der Natur und Berufung stammenden unterschiedlichen Formen der Realisierung dieser Aufgaben. In einer harmonischen Familie sah sie das Bedürfnis nach der Komplementarität, sowohl der Geschlechter als auch der Generationen. Ihre Vorstellung von Ehe und Familie hat nichts an Aktualität verloren.

\section{Bibliography}

Biberstein - Stein E., Aufzeichnungen, in: E. Stein, Aus dem Leben einer jüdischen Familie, Freiburg 1985.

Frank K. S., Familie. III. Soziologisch, in: Lexikon für Theologie und Kirche, B. 3, Freiburg 2006.

Franziskus, Apostolisches Schreiben Amoris laetitia.

Gerl H.-B., Einleitung, in: E. Stein, Briefe an Roman Ingarden, Freiburg 1991.

Johannes Paul II., Apostolisches Schreiben Familiaris consortio.

Leuven R., Heil im Unheil, Freiburg 1983.

Müller, A. U., Neyer M. A., Edith Stein. Das Leben einer ungewöhnlichen Frau, Zürich 1998.

Sander H.-J., Theologischer Kommentar zur Pastoralkonstitution über die Kirche in der Welt von heute Gaudium et spes, in: Herders Theologischer Kommentar zum Zweiten Vatikanischen Konzil, B. 4, Freiburg 2005.

Stein E., Aus dem Leben einer jüdischen Familie, Freiburg 1985.

\footnotetext{
77 Franziskus, Apostolisches Schreiben Amoris laetitia, 31.
} 
Stein E., Beiträge zur philosophischen Begründung der Psychologie und der Geisteswissenschaften. Individuum und Gemeinschaft, „Jahrbuch für Philosophie und phänomenologische Forschung“ 5, Halle a. d. S. 1922.

Stein E., Beruf des Mannes und der Frau nach Natur - und Gnadenordnung, in: Edith Steins Werke, B. 5, Die Frau. Ihre Aufgabe nach Natur und Gnade, Freiburg 1959, S. 17-44.

Stein E., Briefe an Roman Ingarden, Freiburg 1991.

Stein E., Christliches Frauenleben, in: Edith Steins Werke, B. 5, Die Frau. Ihre Aufgabe nach Natur und Gnade, Freiburg 1959, S. 45-72.

Stein E., Das Ethos der Frauenberufe, in: Edith Steins Werke, B. 5, Die Frau. Ihre Aufgabe nach Natur und Gnade, Freiburg 1959, S. 1-15.

Stein E., Der Eigenwert der Frau in seiner Bedeutung für das Leben des Volkes, in: Edith Steins Werke, B. 5, Die Frau. Ihre Aufgabe nach Natur und Gnade, Freiburg 1959, S. 205-217.

Stein E., Die theoretischen Grundlagen der sozialen Bildungsarbeit, in: Edith Stein Gesamtausgabe, B. 16, Freiburg im Breisgau 2001, S. 15-34.

Stein E., Eine Untersuchung über den Staat, ,Jahrbuch für Philosophie und phänomenologische Forschung" 7, Halle a. d. S. 1925.

Stein E., Endliches und ewiges Sein, Freiburg 1986.

Stein E., Ganzheitliches Leben. Schriften zur religiösen Bildung, Edith Steins Werke, B. 12, Freiburg 1990.

Stein E., Jugendbildung im Licht des katholischen Glaubens. Bedeutung des Glaubens und der Glaubenswahrheiten für Bildungsidee und Bildungsarbeit, in: Edith Stein Gesamtausgabe, B. 16, Freiburg 2001, S. 71-90.

Stein E., Mütterliche Erziehungskunst, in: Edith Stein Gesamtausgabe, B. 13, Freiburg 2002, S. 115-126.

Stein E., Probleme der Frauenbildung, in: Edith Steins Werke, B. 5, Die Frau. Ihre Aufgabe nach Natur und Gnade, Freiburg 1959, S. 93-187.

Stein E., Selbstbildnis in Briefen 1, Freiburg 1998.

Stein E., Selbstbildnis in Briefen 2, Freiburg 1977.

Stein E., Was ist der Mensch? Eine theologische Anthropologie, Freiburg 1994. 